\title{
PULLING BACK THE GROMOLL-MEYER CONSTRUCTION AND MODELS OF EXOTIC SPHERES
}

\author{
L. D. SPERANÇA \\ (Communicated by Guofang Wei)
}

\begin{abstract}
The Gromoll-Meyer construction of an exotic 7-sphere is generalized by producing geometric models of exotic 8-, 10- and Kervaire spheres as quotients of principal bundles over spheres. Also, using the geometry of their geodesics, the group structure of homotopy 8-spheres is explicitly presented.
\end{abstract}

\section{INTRODUCTION}

An exotic sphere is a differentiable manifold homeomorphic, but not diffeomorphic, to the standard Euclidean sphere $S^{n}$. The existence of such manifolds was discovered by Milnor [Mil56], who described some 7-dimensional exotic spheres as 3 -sphere bundles over $S^{4}$ with structure group $S O(4)$.

A milestone in the study of their geometry was the presentation, by Gromoll and Meyer GM72, of an exotic sphere as a quotient of the Lie group $S p(2)$ by a simple $S^{3}$-action. More precisely, considering $S^{7}$ as the unitary sphere of the quaternionic plane $\mathbb{H}^{2}$ and

$$
S p(2)=\left\{Q=\left(\begin{array}{ll}
\eta_{1} & \eta_{3} \\
\eta_{2} & \eta_{4}
\end{array}\right) \in S^{7} \times S^{7} \mid \bar{\eta}_{3} \eta_{1}+\bar{\eta}_{4} \eta_{2}=0\right\},
$$

then the group of unit quaternions $S^{3}$ acts in $S p(2)$ by

$$
q \star\left(\begin{array}{ll}
\eta_{1} & \eta_{3} \\
\eta_{2} & \eta_{4}
\end{array}\right)=\left(\begin{array}{ll}
q \eta_{1} \bar{q} & q \eta_{3} \\
q \eta_{2} \bar{q} & q \eta_{4}
\end{array}\right)
$$

Its quotient is a generator of the cyclic group of homotopy 7-spheres (see EK62]).

This construction fits inside a richer framework. First observe that we have a "standard" action of $S^{3}$ in $S p(2)$ :

$$
q \bullet\left(\begin{array}{ll}
\eta_{1} & \eta_{3} \\
\eta_{2} & \eta_{4}
\end{array}\right)=\left(\begin{array}{ll}
\eta_{1} & \eta_{3} \bar{q} \\
\eta_{2} & \eta_{4} \bar{q}
\end{array}\right)
$$

Received by the editors May 29, 2014 and, in revised form, August 23, 2014, March 26, 2015 and September 1, 2015.

2010 Mathematics Subject Classification. Primary 57R60, 57R50.

The author was financially supported by FAPESP, grant numbers 2009/07953-8 and 2012/25409-6. 
Actions (1.1) and (1.2) commute, giving rise to a cross diagram,

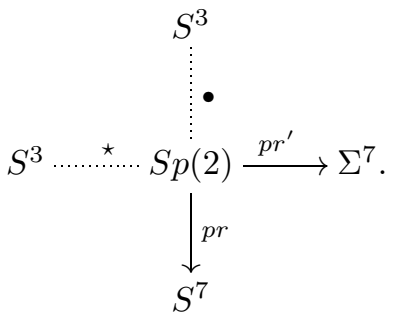

This diagram contains a wealth of information about the geometry of the Gromoll-Meyer sphere, especially with respect to its geodesics and submanifolds (e.g. ADPR07,Dur01,DMR04,DR09]). For instance, note that each one of the actions descends to symmetries of the quotient by the other action.

The main point of this paper is that the Gromoll-Meyer construction, understood as diagram (1.3), can be pulled back to give explicit quotient models of other exotic spheres. The main results are the following theorems.

Theorem 1. There are explicit principal $S^{3}$-bundles $\pi^{11}: E^{11} \rightarrow S^{8}$ and $\pi^{13}$ : $E^{13} \rightarrow S^{10}$ such that

(1) $E^{11}$ admits an explicit free $S^{3}$-action which commutes with the principal one and whose quotient is the only exotic 8-sphere;

(2) $E^{13}$ admits an explicit free $S^{3}$-action which commutes with the principal one and whose quotient is a generator of the order 3 group of homotopy 10-spheres which bound spin manifolds.

We remark that all of the above actions are isometric for a big family of KaluzaKlein metrics (Lemma 4.3).

Pullback constructions related to exotic spheres first appeared in Wil01, where iterated pullbacks of the Gromoll-Meyer construction were used to produce $\left(S^{3}\right)^{m}$ manifolds whose quotients were diffeomorphic to the bundles in Mil56]. Further, in DPR10 a pullback construction was used to produce all 7-dimensional exotic spheres. To the author's knowledge, the present paper is the first application of this kind of construction for homotopy spheres not bounding parallelizable manifolds.

We also show that these ideas can be applied to bundles unrelated to the GromollMeyer construction. In Section 5, we present all Kervaire manifolds as quotients of $O(n)$-actions on pullbacks of frame bundles of round spheres.

Theorem 2. For every $n \in \mathbb{N}$, there exists an explicit principal $O(n)$-bundle $\pi_{n}: L_{n} \rightarrow S^{2 n-1}$ which admits an explicit free $O(n)$-action, commuting with the principal one, whose quotient is the Kervaire manifold $K^{2 n-1}$.

The $S^{3}$-manifolds in DPR10, Wil01 and the $O(n)$-manifold $K^{2 n-1}$ are equivariantly diffeomorphic to the ones considered in Dav82 and $\partial P^{2 n}\left(A_{2}\right)$ in Bre72, Section V.8], where they are classified up to equivariant diffeomorphism type.

As geometric applications, we pull back to our examples the Wiedersehen metrics and their relationship with exotic diffeomorphisms that began in Dur01. There, an explicit Wiedersehen metric on the Gromoll-Meyer sphere is used to construct a rather symmetric formula for an exotic diffeomorphism (degree 1 but not isotopic to the identity) $\sigma: S^{6} \rightarrow S^{6}$. If one thinks of homotopy $n$-spheres as manifolds 
obtained by gluing two discs by a diffeomorphism of their boundary (as Smale's theorem allows for $n>4$ ), the group structure given by connected sum of homotopy $n$-spheres is translated to composition in the group of isotopy classes of diffeomorphisms of the standard $(n-1)$-sphere. Here we produce a clutching diffeomorphism of the exotic 8-sphere, together with an isotopy of its square to the identity (thus realizing the group of homotopy 8-spheres as the cyclic group with two elements).

1.1. Notational conventions. Throughout this paper, we consider what we call a special $G-G$ bundle (or $G-G$ bundle for short; compare Las82]): a $G$-principal bundle $\pi: P \rightarrow M$, where $P$ is a $G \times G$-manifold, $M$ is a $G$-manifold, $\pi$ is principal with respect to the $G$-action defined by $\{1\} \times G$ and equivariant with respect to the one defined by $G \times\{1\}$; furthermore, we require that $G \times\{1\}$ acts freely, denoting its quotient by $M^{\prime}$ and its quotient map by $\pi^{\prime}: P \rightarrow M^{\prime}$.

Given a $G$ - $G$ bundle $\pi: P \rightarrow M$, we call the $G$-actions defined by $\{1\} \times G$ and $G \times\{1\}$ as the $\bullet$-action and the $\star$-action, respectively. We denote the $\bullet$-action by right juxtaposition, denoting by $s \in G$ the acting element, and both the $\star$ and the $G$-action on $M$ by left juxtaposition by $q \in G$, i.e., if $p \in P$, the $\bullet$-action of an arbitrary element of $G$ will be denoted by $p s^{-1}$ (the inverse is used to maintain the left-action convention) and the $\star$-action as $q p$. We use the same pattern for $S p(2)$, denoting action (1.2) by $Q s^{-1}$ and (1.1) by $q Q$.

With this convention, the equivariance of $\pi$ reads $\pi(q p)=q \pi(p)$. We also observe that the $G$-action on $M$ is completely determined by the $\star$-action on $P$. Analogously, $M^{\prime}$ has a unique $G$-action descending from the $\bullet$-action on $P$. This is the unique action that makes $\pi^{\prime}$ a $G-G$ bundle.

Our preference for considering $G-G$ bundles instead of $G \times G$-manifolds is based on the various papers ADPR07, Dur01, DMR04, DP09, DRS10, which suggest that we gain interesting information from the study of $P$ as a bundle over $M$. This is illustrated throughout the paper, especially in Sections 3 and 4.

The symbols $\eta_{1}, \eta_{2}, \eta_{3}, \eta_{4}, w_{1}, w_{2}$ will denote elements of the quaternionic field $\mathbb{H}$ and $\xi$ an element of the subspace of pure imaginary quaternions $\operatorname{Im} \mathbb{H}$. $\lambda$ is a real number. Juxtaposition of quaternions will always mean quaternionic multiplication.

We often consider products of manifolds; more specifically, products of discs with spheres, spheres with spheres, and manifolds with groups. We denote arbitrary elements on products of discs with spheres and spheres with spheres as $\left(x_{1}, x_{2}\right)$. For products of manifolds and groups (which are usually related to trivializations of bundles), we always denote the group element as $g$ (even if it is a quaternion).

The letter $p$ is always a point of $P$ and $x^{\prime}$ of $M^{\prime}$. We will use $x$ for elements in $M$ or in subsets of $M$; we use $x \in D^{7} \subset S^{7}$, for example. The only exception is $S p(2)$, where we denote an arbitrary element as $Q$.

\section{Pulling Back the Gromoll-Meyer construction to 8- And 10-DIMENSIONAL EXOTIC SPHERES}

Consider, as in the Introduction, the Gromoll-Meyer construction as a pair of commuting actions on $S p(2)$. We present here a natural procedure to pull back both actions simultaneously. We first describe it in a general way and then specialize to the cases in Theorem 1 
We start by observing that a quotient map for action (1.2) is $p r: S p(2) \rightarrow S^{7}$, the map that sends a matrix in $S p(2)$ to its first column,

$$
\operatorname{pr}\left(\left(\begin{array}{ll}
\eta_{1} & \eta_{3} \\
\eta_{2} & \eta_{4}
\end{array}\right)\right)=\left(\begin{array}{l}
\eta_{1} \\
\eta_{2}
\end{array}\right)
$$

There is a unique $S^{3}$-action on $S^{7}$ that makes it equivariant with respect to (1.1):

$$
q\left(\begin{array}{l}
\eta_{1} \\
\eta_{2}
\end{array}\right)=\left(\begin{array}{c}
q \eta_{1} \bar{q} \\
q \eta_{2} \bar{q}
\end{array}\right)
$$

From now on we consider $S^{7}$ as the $S^{3}$-manifold with action (2.1) and $p r: S p(2) \rightarrow$ $S^{7}$ as the special $S^{3}-S^{3}$ bundle with (1.1) and (1.2).

Given a map $f: M \rightarrow S^{7}$, one can define the pullback bundle $\pi: f^{*} S p(2) \rightarrow M$ as the bundle with total space

$$
f^{*} S p(2)=\{(x, Q) \in M \times S p(2) \mid f(x)=\operatorname{pr}(Q)\}
$$

and projection $\pi(x, Q)=x$. In particular, $(x, Q) \in f^{*} S p(2)$ if and only if it has the form

$$
(x, Q)=\left(x,\left(\begin{array}{ll}
\eta_{1}(x) & \eta_{3} \\
\eta_{2}(x) & \eta_{4}
\end{array}\right)\right)
$$

where $\eta_{1}$ and $\eta_{2}$ are defined by $\left(\eta_{1}(x), \eta_{2}(x)\right)^{t}=f(x) \in S^{7}$. Equivalently, (up to isomorphism) $f^{*} S p(2)$ is the only principal $S^{3}$-bundle that fits in the "pullback diagram"

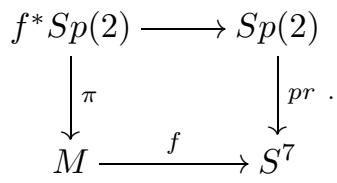

Here the upper horizontal arrow is the projection in the second coordinate.

As a subspace, $f^{*} S p(2)$ is naturally a $G$-principal bundle over $M$ with action $(x, Q) s^{-1}=\left(x, Q s^{-1}\right)$. It inherits extra structures according to the properties of $f$. For instance, it inherits a $\star$-action if we require equivariance: suppose that $M$ is an $S^{3}$-manifold and that $f: M \rightarrow S^{7}$ is a $S^{3}$-equivariant map; then $f^{*} S p(2)$ admits an induced $\star$-action defined by the formula

$$
q(x, Q)=(q x, q Q) .
$$

It is free, since it is free in the $S p(2)$-coordinate, and well defined, since $\operatorname{pr}(q Q)=$ $f(q x)$, thus defining $f^{*} S p(2) \stackrel{\pi}{\rightarrow} M$ as a $S^{3}-S^{3}$ bundle.

In what follows, we produce examples where $M$ is a Euclidean sphere and $M^{\prime}$ is an exotic one. We use their geometry to relate different presentations of $M^{\prime}$.

Remark 2.1. It is worth mentioning that the differentiable class of $f^{*} S p(2) /\left(S^{3} \times\right.$ $\{1\}$ ) is invariant under equivariant homotopies of $f$. This follows from the equivariant version of the classical isomorphism between pullbacks induced by homotopic maps (see [Las82, Corollary 2.11]), and also reflects the properties of the reentrance procedure discussed in Section 3.1. In particular, the geometrically non-appealing map (2.5), used to define $\pi^{11}$, could be replaced by

$$
\tilde{f}_{8}\left(\begin{array}{c}
\lambda \\
w_{1} \\
w_{2}
\end{array}\right)=\frac{1}{\sqrt{\lambda^{2}+\left|w_{1}\right|^{4}+\left|w_{2}\right|^{2}}}\left(\begin{array}{c}
\lambda+w_{1} i \bar{w}_{1} \\
w_{2}
\end{array}\right) .
$$


In contrast to Wil01, we do not use submersions as pullback maps, but topologically motivated, highly singular maps. These maps preserve different geometrical properties, namely, the geodesic joins $S^{8}=S^{4} * S^{3}$ and $S^{10}=S^{6} * S^{3}$ (compare [DP09]).

2.1. 8- and 10-dimensional spheres. Consider $S^{8}$ as the unit sphere in $\mathbb{R} \times \mathbb{H}^{2}$ and define $f_{8}: S^{8} \rightarrow S^{7}$ as

$$
f_{8}\left(\begin{array}{c}
\lambda \\
w_{1} \\
w_{2}
\end{array}\right)=\left(\begin{array}{c}
\sqrt{1-\varphi\left(\left|w_{2}\right|\right)^{2}} \frac{\lambda+w_{1} i \bar{w}_{1}}{\sqrt{\lambda^{2}+\left|w_{1}\right|^{4}}} \\
\varphi\left(\left|w_{2}\right|\right) \frac{w_{2}}{\left|w_{2}\right|}
\end{array}\right)
$$

where $\varphi$ is a smooth non-decreasing function that is the identity on $\left[\frac{1}{4}, \frac{3}{4}\right]$ and constant near 0 and 1 , fixing 0 and 1 .

We endow $S^{8}$ with the following $S^{3}$-action,

$$
q\left(\begin{array}{c}
\lambda \\
w_{1} \\
w_{2}
\end{array}\right)=\left(\begin{array}{c}
\lambda \\
q w_{1} \\
q w_{2} \bar{q}
\end{array}\right)
$$

thus making $f_{8}$ equivariant with respect to (2.1). In particular, it induces a special $S^{3}-S^{3}$ bundle $\pi^{11}: E^{11}=f_{8}^{*} S p(2) \rightarrow S^{8}$ whose quotient by the $\star$-action we denote $\Sigma^{8}$. This action is written as

$$
q\left(\begin{array}{c}
\lambda \\
w_{1} \\
w_{2}
\end{array}\left(\begin{array}{ll}
\eta_{1}(x) & \eta_{3} \\
\eta_{2}(x) & \eta_{4}
\end{array}\right)\right)=\left(\begin{array}{c}
\lambda \\
q w_{1} \\
q w_{2} \bar{q}
\end{array}\left(\begin{array}{cc}
q \eta_{1}(x) \bar{q} & q \eta_{3} \\
q \eta_{2}(x) \bar{q} & q \eta_{4}
\end{array}\right)\right) .
$$

For the 10-dimensional case, consider $S^{10}$ as the unit sphere in $\operatorname{Im} \mathbb{H} \times \mathbb{H}^{2}$ and endow it with the $S^{3}$-action

$$
q\left(\begin{array}{c}
\xi \\
w_{1} \\
w_{2}
\end{array}\right)=\left(\begin{array}{c}
\xi \\
q w_{1} \\
q w_{2} \bar{q}
\end{array}\right)
$$

with $\xi \in \operatorname{Im} \mathbb{H}$ and $w_{1}, w_{2} \in \mathbb{H}$. Define the smooth equivariant map $f_{10}: S^{10} \rightarrow S^{7}$ by

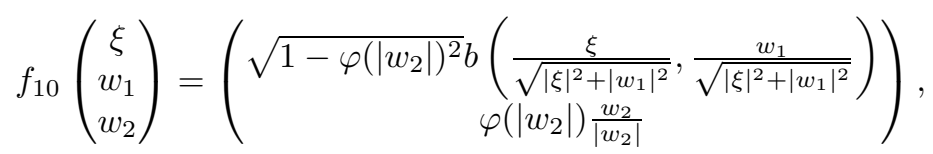

where $\varphi$ is as in (2.5) and $b: S^{6} \rightarrow S^{3}$ is the analytic transition map for $p r$ defined in DMR04:

$$
b\left(\xi, w_{1}\right)= \begin{cases}\frac{w_{1}}{\left|w_{1}\right|} e^{\pi \xi} \frac{\bar{w}_{1}}{\left|w_{1}\right|}, & w_{1} \neq 0 \\ -1, & w_{1}=0 .\end{cases}
$$

Pulling back by $f_{10}$ gives a new special $S^{3}-S^{3}$ bundle $\pi^{13}: f_{10}^{*} S p(2)=E^{13} \rightarrow S^{10}$ whose quotient by the $\star$-action we denote $\Sigma^{10}$. These are the bundles in Theorem 1 . We recognize the differentiable structures of $\Sigma^{8}$ and $\Sigma^{10}$ in the next section by identifying them as the plumbing manifolds in Sch72]. In Section 4, we apply ideas from Dur01 to get explicit clutching diffeomorphisms and the desired isotopy.

Remark 2.2. Although we use smooth representatives of $f_{8}$ and $f_{10}$ throughout the text, we can safely replace $\varphi$ by the identity, which simplifies the proofs. In fact, complementing Remark 2.1 even a non-smooth continuous equivariant map 
$f: M \rightarrow S^{7}$ defines a unique differentiable structure on $f^{*} S p(2) /\left(S^{3} \times\{1\}\right)$ : one can equivariantly smooth $f$ and recall that two smooth equivariant representatives are smoothly equivariant (compare [DMR04, pp. 5036-5042] where differentiability issues are carefully studied). A very simple application of this principle is used in Theorem 4.6 .

In general, given a special $G-G$ bundle $P \rightarrow M$ and another $G$-manifold $N$, the pullback construction furnishes a well defined map from the space of homotopy classes of equivariant continuous maps from $N$ to $M$ to the space of equivariant diffeomorphism classes of smooth $G$-manifolds. This relates homotopy classes of equivariant maps with equivariant diffeomorphism invariants.

\section{ReCOGNIZING the EXOTIC SPHERES}

A quotient model eventually gives more than the possibility to work without coordinates, it also gives greater intuition on the structure of the manifold: choosing different local sections on the total space gives different decompositions of the quotient. Here we get different descriptions of our quotients using the pullback nature of their total spaces and relate the following constructions:

(1) geometric quotients (Theorems 1 and 2);

(2) Milnor's plumbing construction ([Mil59A], Theorem 3.2);

(3) the gluing of two discs by an "exotic" diffeomorphism (Theorem 4.6).

Recognizing $\Sigma^{8}$ and $\Sigma^{10}$ as plumbing manifolds allows the classification of their differentiable classes. This is done by pulling back a plumb-like trivialization of $S p(2)$. In 3.1 we begin by describing the $S^{3}-S^{3}$ bundle structure of $p r$ and give a plumbing description of the Gromoll-Meyer sphere. Then we pull it back to $\Sigma^{8}$ and $\Sigma^{10}$ in 3.2 .

3.1. $S p(2) \rightarrow S^{7}$ as a special $S^{3}-S^{3}$ bundle. Since $p r$ is a bundle over a sphere, $S p(2)$ can be obtained by gluing two copies of $D^{7} \times S^{3}$ around their boundaries. According to DMR04, a gluing diffeomorphism for this presentation is $f_{b}(x, g)=(x, g b(x))$, with $b$ as in (2.10). The geometry of the trivializations in Dur01,DMR04 gives the map $b$ a special type of equivariance, which we call conjugation equivariance: for every $x \in S^{6}$ and $q \in S^{3}$,

$$
b(q x)=q b(x) q^{-1},
$$

where $q x=\left(q \xi \bar{q}, q w_{2} \bar{q}\right) \in \operatorname{Im} \mathbb{H} \times \mathbb{H},|\xi|^{2}+\left|w_{2}\right|^{2}=1$. This property can be seen as the reason for the existence of action (1.1). In fact, in the trivializations of [Dur01, action (1.1) is written (locally, in each copy of $D^{7} \times S^{3}$ ) as

$$
q(x, g)=\left(q x, g q^{-1}\right) .
$$

This formula defines a global action on the total space if and only if $b$ satisfies (3.1). Furthermore, (3.2) has a very explicit quotient map:

$$
\begin{aligned}
\pi^{\prime}: D^{7} \times S^{3} & \rightarrow D^{7}, \\
(x, g) & \mapsto g x .
\end{aligned}
$$

Indeed, $\pi^{\prime}$ is smooth, $\pi^{\prime}\left(q x, g q^{-1}\right)=\pi^{\prime}(x, g)$, and $D^{7} \times\{1\}$ is a section, therefore, each copy of $D^{7} \times S^{3}$ descends to a disc via $\pi^{\prime}$. The gluing diffeomorphism of these discs is explicitly calculated via

$$
\pi^{\prime}\left(f_{b}(x, 1)\right)=b(x) \pi^{\prime}(x, 1),
$$


where $b(x)$ acts on $\pi^{\prime}(x, 1)$ via (2.1) and $f_{b}$ was defined above. It follows that $x \mapsto b(x) x$ is a clutching diffeomorphism for the Gromoll-Meyer sphere.

The merit of this procedure lies in its generality (compare [DRS10]). We obtained this clutching diffeomorphism using quite general facts: let $P \rightarrow M$ be a $G$ - $G$ bundle and $\Psi_{+}, \Psi_{-}: U_{ \pm} \times G \rightarrow P$ be local trivializations compatible with (3.2), i.e.,

$$
\Psi_{ \pm}\left(q x, s g q^{-1}\right)=q \Psi_{ \pm}(x, g) s^{-1} .
$$

The projection analogous to (3.3) identifies $\pi^{-1}\left(U_{ \pm}\right)$with $U_{ \pm}$. Denoting the associated transition function by $t: U_{+} \cap U_{-} \rightarrow G$, we have

$$
\pi^{\prime}\left(\Psi_{+}(x, 1) t(x)^{-1}\right)=t(x) \pi^{\prime}\left(\Psi_{-}(x, 1)\right) .
$$

Observing that $\Psi_{ \pm}\left(U_{ \pm} \times\{1\}\right)$ are sections, we conclude that $\pi^{\prime}\left(\pi^{-1}\left(U_{+} \cup U_{-}\right)\right)$is equivariantly diffeomorphic to $U_{+} \cup_{\hat{t}} U_{-}$, where $\hat{t}(x)=t(x) x$.

This observation suggests the procedure introduced in [DRS10] as "the reentrance procedure" (see [Bre72, I.6 (C)] as well): given a $G$-manifold $X$ and a smooth map $t: X \rightarrow G$ satisfying (3.1), we can define the diffeomorphism

$$
\begin{aligned}
\hat{t}: X & \rightarrow X, \\
x & \mapsto t(x) x .
\end{aligned}
$$

We call $\hat{t}$ the reentrance associated to $t$. The association $t \mapsto \hat{t}$ is an antihomomorphism from the group of conjugation equivariant smooth maps with pointwise multiplication to the group of $G$-equivariant diffeomorphisms of $X$. That is, if $t, r$ are maps as above, then $\widehat{t r}=\hat{r} \hat{t}$ where $(t r)(x)=t(x) r(x)$. Moreover, equivariant homotopies of $t$ produce isotopies of $\hat{t}$ (see [DR09]). These facts are used throughout the text, specifically in Theorem 4.6 .

We just observed that quotients of $\star$-actions are completely determined by the reentrance of transition functions. In order to identify the differentiable classes of $\Sigma^{8}$ and $\Sigma^{10}$, we choose the sets $U_{ \pm}$to induce plumbing-like decompositions on them.

Let us begin describing a plumbing in a geometric way. Observe that one can produce a 7 -sphere by identifying the common boundaries of $D^{4} \times S^{3}$ and $S^{3} \times D^{4}$. We do it by first considering the following subsets of $S^{7}$,

$$
\begin{aligned}
& \nu\left(S^{3}\right)_{-}=\left\{\left(\eta_{1}, \eta_{2}\right) \in S^{7} \mid \eta_{2} \neq 0\right\}, \\
& \nu\left(S^{3}\right)_{+}=\left\{\left(\eta_{1}, \eta_{2}\right) \in S^{7} \mid \eta_{1} \neq 0\right\} .
\end{aligned}
$$

These subsets intersect on a tubular neighborhood of $|x|^{2}=|y|^{2}=1 / 2$. Thus $S^{7}$ is obtained by gluing them along their intersection, which is diffeomorphic to $S^{3} \times S^{3} \times(0,1)$. We still get the same topological space if we discard "half" of the intersection, replacing $\nu\left(S^{3}\right)_{ \pm}$by the subsets,

$$
\begin{aligned}
& \bar{\nu}\left(S^{3}\right)_{-}=\left\{\left.\left(\eta_{1}, \eta_{2}\right) \in S^{7}|| \eta_{2}\right|^{2} \geq 1 / 2\right\}, \\
& \bar{\nu}\left(S^{3}\right)_{+}=\left\{\left.\left(\eta_{1}, \eta_{2}\right) \in S^{7}|| \eta_{1}\right|^{2} \geq 1 / 2\right\} .
\end{aligned}
$$

Since (3.9) and (3.10) are diffeomorphic to $D^{4} \times S^{3}$ and $S^{3} \times D^{4}$, respectively, we obtain the desired description of $S^{7}$. Since there were no twists in the gluing, this defines $S^{7}$ as the "trivial" plumbing.

In spite of losing open charts when we consider the gluing by non-open subsets, we still insist on calling the plumbing a differentiable manifold. This possibility 
has already been observed by Milnor in [Mil59B, Appendix] since the last identification can be done in a unique way in the differentiable category: there is only one differentiable structure in the resulting space that preserves the differentiable structures of the products of discs with spheres and the isotopy class of the gluing diffeomorphism (which is the identity id : $S^{3} \times S^{3} \rightarrow S^{3} \times S^{3}$ in this case). In other words, there is only one way to "straighten the corners".

The next step is to give $S p(2)$ a similar structure.

Lemma 3.1. $S p(2)$ with the $\bullet$ and $\star$ actions is equivariantly diffeomorphic to

$$
D^{4} \times S^{3} \times S^{3} \cup_{f_{\theta_{7}}} S^{3} \times D^{4} \times S^{3},
$$

where $f_{\theta_{7}}\left(x_{1}, x_{2}, g\right)=\left(x_{1}, x_{2}, g x_{1} \bar{x}_{2}\right)$ and, in each part, the $\bullet$ and $\star$ actions are

$$
q\left(x_{1}, x_{2}, g\right) s^{-1}=\left(q x_{1} \bar{q}, q x_{2} \bar{q}, s g \bar{q}\right) .
$$

Proof. Let us use the subsets $\bar{\nu}\left(S^{3}\right)_{ \pm}$to trivialize $S p(2)$ : define $\Psi_{-}: D^{4} \times S^{3} \times S^{3} \rightarrow$ $S p(2)$ and $\Psi_{+}: S^{3} \times D^{4} \times S^{3} \rightarrow S p(2)$ by

$$
\begin{aligned}
& \Psi_{-}\left(\begin{array}{c}
x_{1} \\
x_{2} \\
g
\end{array}\right)=\left(\begin{array}{cc}
\sin \left(\frac{\pi\left|x_{1}\right|}{4}\right) \frac{x_{1}}{\left|x_{1}\right|} & -\cos \left(\frac{\pi\left|x_{1}\right|}{4}\right) \bar{g} \\
\cos \left(\frac{\pi\left|x_{1}\right|}{4}\right) x_{2} & \sin \left(\frac{\pi\left|x_{1}\right|}{4}\right) \frac{x_{2} \bar{x}_{1}}{\left|x_{1}\right|} \bar{g}
\end{array}\right), \\
& \Psi_{+}\left(\begin{array}{c}
x_{1} \\
x_{2} \\
g
\end{array}\right)=\left(\begin{array}{cc}
\cos \left(\frac{\pi\left|x_{2}\right|}{4}\right) x_{1} & -\sin \left(\frac{\pi\left|x_{2}\right|}{4}\right) \frac{x_{1} \bar{x}_{2}}{\left|x_{2}\right|} \bar{g} \\
\sin \left(\frac{\pi\left|x_{2}\right|}{4}\right) \frac{x_{2}}{\left|x_{2}\right|} & \cos \left(\frac{\pi\left|x_{2}\right|}{4}\right) \bar{g}
\end{array}\right) .
\end{aligned}
$$

To compute the transition function along $S^{3} \times S^{3} \times\{1\}$, we set $\left|x_{1}\right|=\left|x_{2}\right|=1$ and get $\Psi_{-}\left(x_{1}, x_{2}, 1\right)=\Psi_{+}\left(x_{1}, x_{2}, 1\right) x_{2} \bar{x}_{1}=\Psi_{+}\left(f_{\theta_{7}}\left(x_{1}, x_{2}, 1\right)\right)$.

Here $\theta_{7}: S^{3} \times S^{3} \rightarrow S^{3}$, defined by $\theta_{7}\left(x_{1}, x_{2}\right) \mapsto x_{1} \bar{x}_{2}$, is the transition map associated to the trivializations (3.11) and (3.12). These trivializations not only endow $S p(2)$ with a plumbing-like decomposition, but also descend to $\Sigma^{7}$ as a genuine plumbing presentation: $\Sigma^{7}=D^{4} \times S^{3} \cup_{g f} S^{3} \times D^{4}$ where $f\left(x_{1}, x_{2}\right)=$ $\left(x_{1}, x_{1} x_{2} \bar{x}_{1}\right)$ and $g\left(x_{1}, x_{2}\right)=\left(x_{2} x_{1} \bar{x}_{2}, x_{2}\right)$. The form of the transition maps and their relation to plumbing will be preserved by the pullback procedure, as we shall see in the next section.

3.2. The 8- and 10-dimensional cases. Using standard facts on pullback bundles (as in [Ste99, Section 10.1]), we get plumbing-like descriptions for $\pi^{11}$ and $\pi^{13}$. Note that the $f_{8}$-preimages of the subspaces $\bar{\nu}\left(S^{3}\right)_{ \pm}$are the trivializing subsets,

$$
\begin{aligned}
& f_{8}^{-1}\left(\bar{\nu}\left(S^{3}\right)_{-}\right)=\left\{\left(\lambda, w_{1}, w_{2}\right) \in S^{8} \mid w_{2} \geq 1 / 2\right\}, \\
& f_{8}^{-1}\left(\bar{\nu}\left(S^{3}\right)_{+}\right)=\left\{\left.\left(\lambda, w_{1}, w_{2}\right) \in S^{8}\left|\lambda^{2}+\right| w_{1}\right|^{2} \geq 1 / 2\right\},
\end{aligned}
$$

thus giving a description of $S^{8}$ as the gluing of $D^{5} \times S^{3}$ and $S^{4} \times D^{4}$ whose intersection is exactly the preimage of $\bar{\nu}\left(S^{3}\right)_{+} \cap \bar{\nu}\left(S^{3}\right)_{-}$. In this description, the transition map of $\pi^{11}$ associated with this trivializations is

$$
\begin{aligned}
& \theta_{8}: S^{4} \times S^{3} \rightarrow S^{3}, \\
& \left(\left(\lambda, w_{1}\right), w_{2}\right) \mapsto \eta\left(\lambda, w_{1}\right) w_{2}^{-1},
\end{aligned}
$$

where $\eta: S^{4} \rightarrow S^{3}$ is a suspended Hopf map

$$
\eta\left(\lambda, w_{1}\right)=\frac{\lambda+w_{1} i \bar{w}_{1}}{\sqrt{\lambda^{2}+\left|w_{1}\right|^{4}}} .
$$


Applying an analogous procedure to $\pi^{13}$, we get the transition function $\theta_{10}\left(\left(\xi, w_{1}\right), w_{2}\right)=b\left(\xi, w_{1}\right) \bar{w}_{2}$ defined on $S^{6} \times S^{3}$.

Both $\theta_{8}$ and $\theta_{10}$ factor pointwise as products: $\theta_{n}\left(x_{1}, x_{2}\right)=\alpha_{n}\left(x_{1}\right) \beta_{n}\left(x_{2}\right)^{-1}$, where $\alpha_{8}=\eta: S^{4} \rightarrow S^{3}, \alpha_{10}=b: S^{6} \rightarrow S^{3}$, and $\beta_{8}=\beta_{10}=$ id $: S^{3} \rightarrow S^{3}$. All maps are conjugation equivariant with respect to the actions induced from (2.6), (2.8), or quaternionic conjugation.

The next three results - Theorem [3.2, Proposition 3.3, and Corollary 3.5 are valid in the general context of equivariant maps: in all proofs one can consider arbitrary linear $S^{3}$-actions on arbitrary $S^{k}$ and $S^{l}$ and arbitrary smooth conjugation equivariant maps $\alpha: S^{k} \rightarrow S^{3}$ and $\beta: S^{l} \rightarrow S^{3}$ instead of $\alpha_{n}, \beta_{n}$. Proposition 3.3 and Corollary 3.5 are valid even if one replaces $S^{3}$ by an arbitrary group acting linearly on $S^{k}$ and $S^{l}$. The exoticity of $\Sigma^{8}$ and $\Sigma^{10}$ is due to the special homotopy classes one gets combining the actions and maps in the previous paragraph, as we shall see in Corollary 3.5] and in the proof of Theorem 11. This generalization will be used in Section 5 .

Theorem 3.2. The quotient of $E^{n+3}$ by its $\star$-action is diffeomorphic to

$$
\Sigma_{\theta_{n}}=D^{k+1} \times S^{l} \cup_{\widehat{\theta_{n}}} S^{k} \times D^{l+1},
$$

where the action on $S^{k} \times S^{l}$ is the product of the actions on $S^{k}$ and $S^{l}$.

Proof. According to the discussion in 3.1, it is sufficient to find trivializations of $E^{n+3}$ along $f_{n}^{-1}\left(\bar{\nu}\left(S^{3}\right)_{ \pm}\right)$satisfying (3.5) with transition function $\theta_{n}$. This is easily done by pulling back (3.11) and (3.12): using the identifications $\bar{\nu}_{+}\left(S^{3}\right)_{+} \approx S^{3} \times D^{4}$, $\bar{\nu}_{+}\left(S^{3}\right)_{-} \approx D^{4} \times S^{3}, f_{n}^{-1}\left(\bar{\nu}\left(S^{3}\right)_{-}\right) \approx D^{k+1} \times S^{l}$, and $f_{n}^{-1}\left(\bar{\nu}\left(S^{3}\right)_{+}\right) \approx S^{k} \times D^{l+1}$, we write $\tilde{\Psi}_{ \pm}(x, g)=\Psi_{ \pm}\left(f_{n}(x), g\right)$ and conclude that

$$
f_{n}^{*} S p(2)=D^{k+1} \times S^{l} \times S^{3} \cup_{f_{\theta_{n}}} S^{k} \times D^{l+1} \times S^{3},
$$

where $f_{\theta_{n}}(x, y, g)=\left(x, y, g \theta_{n}(x, y)\right)$ and $\tilde{\Psi}_{ \pm}$satisfies (3.6). This is sufficient to prove the theorem.

Our next task is to identify the differentiable classes of $\Sigma_{\theta_{n}}$. To improve readability, we fix $n$ and drop the subindexes of $\theta_{n}, \alpha_{n}$, and $\beta_{n}$. It follows from the next proposition and Remark 3.4 that $\Sigma_{\theta}$ is a plumbing manifold ([Mil59A]).

Proposition 3.3. Let $\hat{\alpha}$ and $\hat{\beta}$ be the reentrance associated to $\alpha$ and $\beta$, then

$$
\hat{\theta}=(\widehat{\alpha} \times \mathrm{id}) \circ g_{\beta}^{-1} f_{\alpha} \circ\left(\mathrm{id} \times \widehat{\beta}^{-1}\right),
$$

where $g_{\beta}, f_{\alpha}: S^{k} \times S^{l} \rightarrow S^{k} \times S^{l}$ are the diffeomorphisms defined by

$$
\begin{aligned}
& g_{\beta}\left(x_{1}, x_{2}\right)=\left(\beta\left(x_{2}\right) x_{1}, x_{2}\right), \\
& f_{\alpha}\left(x_{1}, x_{2}\right)=\left(x_{1}, \alpha\left(x_{1}\right) x_{2}\right) .
\end{aligned}
$$

Proof. Let $A, B: S^{k} \times S^{l} \rightarrow S^{3}$ be the equivariant maps defined by $A(x, y)=\alpha(x)$ and $B(x, y)=\beta(y)$. Then $\hat{A}=f_{\alpha}(\hat{\alpha} \times$ id $)=(\hat{\alpha} \times$ id $) f_{\alpha}$ and $\hat{B}=f_{\beta}(\hat{\beta} \times$ id $)=$ $(\hat{\beta} \times$ id $) f_{\beta}$. Furthermore,

$$
\begin{aligned}
\hat{\theta}=\hat{B}^{-1} \hat{A} & =g_{\beta}^{-1}\left(\mathrm{id} \times \hat{\beta}^{-1}\right)(\hat{\alpha} \times \mathrm{id}) f_{\alpha} \\
& =g_{\beta}^{-1}(\hat{\alpha} \times \mathrm{id})\left(\mathrm{id} \times \hat{\beta}^{-1}\right) f_{\alpha} .
\end{aligned}
$$


However, $g_{\beta}^{-1}(\hat{\alpha} \times \mathrm{id})=(\hat{\alpha} \times \mathrm{id}) g_{\beta}^{-1}$ and $\left(\mathrm{id} \times \hat{\beta}^{-1}\right) f_{\alpha}=f_{\alpha}\left(\mathrm{id} \times \hat{\beta}^{-1}\right)$ since,

$$
f_{\alpha}^{-1}\left(\mathrm{id} \times \hat{\beta}^{-1}\right) f_{\alpha}\left(x_{1}, x_{2}\right)=\left(x_{1}, \alpha\left(x_{1}\right)^{-1} \hat{\beta}^{-1}\left(\alpha\left(x_{1}\right) x_{2}\right)\right)=\left(x_{1}, \hat{\beta}^{-1}\left(x_{2}\right)\right),
$$

from the equivariance of $\hat{\beta}$. An analogous calculation holds for $g_{\beta}^{-1}(\hat{\alpha} \times \mathrm{id})$.

Remark 3.4. $\Sigma_{\theta}$ is diffeomorphic to the plumbing defined by $g_{\beta}^{-1} f_{\alpha}$ since $\hat{\alpha} \times$ id (respectively id $\times \hat{\beta}^{-1}$ ) extends smoothly to $S^{k} \times D^{l+1}$ (respectively $D^{k+1} \times S^{l}$ ).

To identify their differential structures, observe that the linear $S^{3}$ actions on $S^{k}$ and $S^{l}$ are defined through group homomorphisms which we denote $\Delta_{1}: S^{3} \rightarrow$ $S O(k+1)$ and $\Delta_{2}: S^{3} \rightarrow S O(l+1)$. We have

Corollary 3.5. $\Sigma_{\theta}$ is diffeomorphic to the plumbing of $\Delta_{2} \alpha$ and $\Delta_{1} \beta$.

The differentiable class of the plumbing manifolds defined by these elements are already identified in the literature. According to Sch72, the exotic 8-sphere can be obtained by plumbing a generator of $\pi_{3} S O(5)$ with a generator of $\pi_{4} S O(3)$ composed with the standard inclusion $S O(3) \hookrightarrow S O(4)$. The spin bordant exotic 10-spheres are obtained by plumbing a generator of $\pi_{3} S O(7)$ with one of $\pi_{6} S O(3)$ composed with $S O(3) \hookrightarrow S O(4)$. We conclude the proof by identifying $\Delta_{2} \alpha_{n}, \Delta_{1} \beta_{n}$ as these elements.

Proof of Theorem 1. On both cases $\Delta_{1}$ generates $\pi_{3} S O(k+1)$ and $\Delta_{2}$ factors as the double cover of $S O(3)$ followed by the standard inclusion $S O(3) \hookrightarrow S O(4)$. In particular, $\Delta_{1} \beta_{8}$ (resp. $\left.\Delta_{1} \beta_{10}\right)$ is a generator of $\pi_{3} S O(5)$ (resp. $\left.\pi_{3} S O(7)\right)$. The map $\Delta_{2} \alpha_{8}$ (resp. $\Delta_{2} \alpha_{10}$ ) is the composition of a generator of $\pi_{4} S O(3)$ (resp. $\left.\pi_{6} S O(3)\right)$ with the inclusion $S O(3) \hookrightarrow S O(4)$. As discussed above, the plumbing manifolds defined by these elements are the desired ones.

\section{WIEDERSEHEN METRICS AND EXOTIC DIFFEOMORPHISMS}

It is known ([Bes78] $)$ that the behaviour of the geodesics in the round sphere is of a very special kind. It motivates the following definition: a point $x \in M$ in a Riemannian manifold is called Blaschke if its cut locus is at constant distance from $x$. It is Wiedersehen if, in addition, its cut locus is a point. We call a manifold admitting a Blaschke (Wiedersehen) point as a pointed Blaschke (Wiedersehen) manifold. Its topological structure is well known.

Theorem 4.1 (Weinstein, Allamigeon-Warner, Bes78]). A manifold $M^{n}$ is a pointed Blaschke manifold, if and only if $M$ is diffeomorphic to $D \cup_{\sigma} E$ where $D$ is a disc and $E$ is a disc bundle whose boundary is diffeomorphic to $S^{n-1}$ being $\sigma: \partial D^{n} \rightarrow \partial D(\xi)$, a gluing diffeomorphism.

In particular, if $M$ is pointed Wiedersehen, $E$ is the trivial disc bundle over a point and $M$ is a twisted sphere with clutching diffeomorphism $\sigma: S^{n-1} \rightarrow S^{n-1}$.

The construction of a pointed Blaschke metric on such a manifold was carried out by Weinstein ([Bes78] ) using the existence of the gluing diffeomorphism. In Dur01, the process was reverted: starting from an explicit Blaschke metric on the Gromoll-Meyer exotic sphere, a formula for its clutching diffeomorphism was written, inheriting symmetries of the metric. 
In this section, we observe that the method used on Dur01 fits in a more general context. We see that diagram (1.3) works as a translator of "horizontal" geodesics between $M$ and $M^{\prime}$ and prove

Theorem 4.2. Let $P \rightarrow M$ be a special $G-G$ bundle. If $G$ is compact, then $M$ has a fixed point $x$ which is Blaschke (Wiedersehen) for a $G$-invariant metric if and only if $M^{\prime}$ does.

This provides a big family of explicit pointed Wiedersehen metrics on our exotic spheres and also on all exotic 7-spheres in [DPR10]. In the end, we follow ideas in [Dur01,ADPR07, and present some formulas for gluing diffeomorphisms.

An important point in the proof of Theorem 4.2 is the existence of a trivialization around $x$ where the actions are written as (3.2). We prove it through geometric methods (compare Las82]). We start with the following (well-known) technical lemma ([Bre72, Section VI.2]):

Lemma 4.3. Let $\pi: P \rightarrow M$ be a $G-G$ bundle. Then, for any $G$-invariant metric $m$ on $M$, there exists a $G \times G$-invariant metric on $P$ which makes $\pi$ a Riemannian submersion with respect to $m$.

The lemma follows by averaging any connection metric on $\pi$ by the $G \times G$-action.

For the metric in Lemma 4.3, there is a unique metric on $M^{\prime}$ which makes $\pi^{\prime}$ a Riemannian submersion. Its main property, for our purposes, is that geodesics starting orthogonal to $G \times G$-orbits in $P$ stay orthogonal, therefore they descend to geodesics on both $M$ and $M^{\prime}$.

Let $x \in M$ be a point fixed by the action and $p \in \pi^{-1}(x)$. Since $q p \in \pi^{-1}(x)$, for every $q \in G$, there exists a unique $\phi(q)$ such that $q p \phi(q)^{-1}=p$. One may verify that the association $q \mapsto \phi(q)$ defines an automorphism of $G$ whose graph is the isotropy at $p$. That is,

$$
(G \times G)_{p}=\{(q, \phi(q)) \in G \times G \mid q \in G\} .
$$

In particular, the fibers of $\pi$ and $\pi^{\prime}$ coincide at $x$, implying that $x^{\prime}=\pi^{\prime}(p) \in M^{\prime}$ is a fixed point.

For simplicity, we compose the $\bullet$-action with $\phi$ so that $(G \times G)_{p}$ is the diagonal of $G$ in $G \times G$. Any metric invariant by the original $G \times G$-action will be invariant by the new one.

Assuming further that $x \in M$ is Blaschke (Wiedersehen) and denoting its horizontal lift by $\mathcal{L}_{p}: T_{x} M \rightarrow T_{p} P$, we observe that

$$
\pi \exp _{p}^{P}(v)=\exp _{x}^{M}(d \pi v), \quad \pi^{\prime} \exp _{p}^{P}(v)=\exp _{x^{\prime}}^{M^{\prime}}\left(d \pi^{\prime} v\right) .
$$

Theorem 4.2 is a direct consequence of the following proposition.

Proposition 4.4. Let $M$ be as above. If $\exp _{x}^{M}$ is an embedding when restricted to the disc $D \subset T_{x} M$ of radius $\rho$, then $\exp _{x^{\prime}}^{M^{\prime}}$ is an embedding when restricted to the disc $d \pi^{\prime} \mathcal{L}_{p}(D) \subset T_{x^{\prime}} M^{\prime}$ of radius $\rho$.

Proof. Observe that $\exp _{p}^{P}: T_{p} P \rightarrow P$ is a $G \times G$-equivariant map since we chose an invariant metric on $P$. Its composition with $\mathcal{L}_{p}$ defines a map $\Xi: T_{x} M \times G \rightarrow P$,

$$
\Xi(v, g)=\exp _{p}^{P}\left(\mathcal{L}_{p} v\right) g^{-1}=\exp _{p g^{-1}}^{P}\left(\mathcal{L}_{p g^{-1}} v\right) .
$$

Since $\pi(\Xi(v, g))=\exp _{x}^{M}(v)$, the restriction of $\Xi$ to a subset $D \subset T_{x} M$ is a surjective diffeomorphism onto $\pi^{-1}\left(\exp _{x}^{M}(D)\right)$ as far as the restriction $\left.\exp _{x}^{M}\right|_{D}$ is. 
In particular, if $\exp _{x}^{M}$ embeds a disc $D$ of radius $\rho$, so does $\left.\Xi\right|_{D \times\{1\}}$. To see that $\left.\exp _{x^{\prime}}^{M^{\prime}}\right|_{d \pi^{\prime} \mathcal{L}_{p}(D)}$ is an embedding, we note that $\exp _{x^{\prime}}^{M^{\prime}}\left(d \pi^{\prime} \mathcal{L}_{p}(D)\right)=\pi^{\prime} \Xi(D \times\{1\})$ and prove that $\Xi(D \times\{1\})$ is a section for the $\star$-action. We claim:

Lemma 4.5. $\Xi\left(q v, s g q^{-1}\right)=q \Xi(v, g) s^{-1}$. In particular, if $\left.\exp _{x}^{M}\right|_{D}$ is an embedding, $\pi^{-1}\left(\exp _{x}^{M}(D)\right)$ is equivariantly diffeomorphic to $D \times G$ with actions $q(v, g) s^{-1}$ $=\left(q v, s g q^{-1}\right)$.

Proof. It is only left to prove that the $\star$-action has the claimed form. However,

$$
\begin{aligned}
\Xi\left(q v, g q^{-1}\right) & =\exp _{p}^{P}\left(\mathcal{L}_{p} q v\right) q g^{-1}=q \exp _{q^{-1} p}^{P}\left(\mathcal{L}_{q^{-1}}(v)\right) q g^{-1} \\
& =q \exp _{p q^{-1}}^{P}\left(\mathcal{L}_{p q^{-1}}(v)\right) q g^{-1}=q \exp _{p}^{P}\left(\mathcal{L}_{p}(v)\right) q^{-1} q g^{-1} \\
& =q \exp _{p}^{P}\left(\mathcal{L}_{p}(v)\right) g^{-1}=q \Xi(v, g),
\end{aligned}
$$

where the second equality holds from the equivariance of $\exp _{p}^{P}$.

In particular, comparing with section 3.1, we conclude that $D \times\{1\}$ is a section for both actions on $D \times G$. Therefore, $\Xi(D \times\{1\})$ is a section for the $\star$-action on $P$. It is easy to see that the disc $\pi^{\prime} \Xi(D \times\{1\})$ has radius $\rho$ and its complement in $M^{\prime}$ is exactly the cut locus of $x^{\prime}$, since $\overline{\Xi(D \times G)}=\overline{\left(\pi^{\prime}\right)^{-1}\left(\exp _{x^{\prime}}^{M^{\prime}}\left(d \pi^{\prime} \mathcal{L}_{p}(D)\right)\right)}=P$.

Proposition 4.4 provides a rather topological construction of pointed Blaschke (Wiedersehen) metrics on the manifold $M^{\prime}$. In some sense, this process exotifies not only the manifold, but normally a well-understood metric as well.

We now shift our attention to clutching diffeomorphisms of spheres. We do it in a highly implicit way by pulling back the clutching presentation of [3.1] In general, to obtain such a diffeomorphism from a plumbing presentation can be very involved (see Section 3 in [DMR04]), however, in our case this is easily done. In fact, all the algebraic structure, from (3.1) to (3.4), is nicely pulled back to give clutching descriptions in $E^{11}$ and $E^{13}$.

We already saw that the bundle $p r: S p(2) \rightarrow S^{7}$ is isomorphic to

$$
D^{7} \times S^{3} \cup_{f_{b}} D^{7} \times S^{3} \rightarrow S^{7},
$$

where $f_{b}(x, g)=(x, g b(x))$.

Observing that the points $( \pm 1,0) \in S^{7}$ are fixed by the action, we note that the geometric realization of (4.2) is completely described in the present section. The two discs are the north and south hemispheres of $S^{7}$ with respect to the real part of the first coordinate and the equator, where they intersect, is the unit sphere in $\operatorname{Im} \mathbb{H} \times \mathbb{H}$.

In $S^{8}$, the $\lambda$ coordinate defines the hemispheres, with the pulled back equator,

$$
\mathcal{S}^{7}=f_{8}^{-1}\left(S^{6}\right)=\left\{\left(\lambda, w_{1}, w_{2}\right) \in S^{8} \mid \lambda=0\right\},
$$

dividing $S^{8}$ in $S^{3}$-invariant subsets. With these subsets, we realize the bundle $f_{8}^{*} S p(2)$ as

$$
f_{8}^{*} S p(2)=D^{8} \times S^{3} \cup D^{8} \times S^{3},
$$

with clutching function $f_{\Theta_{8}}(x, g)=\left(x, g b\left(f_{8}(x)\right)\right)$ and $\star$-action given as in (3.2) . 
Following Section 3.1, we have

Theorem 4.6. Let $\Theta_{8}: \mathcal{S}^{7} \rightarrow S^{3}$ and $\Theta_{10}: \mathcal{S}^{9} \rightarrow S^{3}$ be defined as

$$
\begin{aligned}
& \Theta_{8}\left(w_{1}, w_{2}\right)= \begin{cases}\frac{w_{2}}{\left|w_{2}\right|} e^{\pi w_{1} i \bar{w}_{1}} \frac{\bar{w}_{2}}{\left|w_{2}\right|}, & w_{2} \neq 0, \\
-1, & w_{2}=0\end{cases} \\
& \Theta_{10}\left(\xi, w_{1}, w_{2}\right)= \begin{cases}b\left(\xi, w_{1}\right) e^{\pi w_{2}} b\left(\xi, w_{1}\right)^{-1}, & w_{2} \neq 0 \\
-1, & w_{2}=0\end{cases}
\end{aligned}
$$

where

$$
\mathcal{S}^{9}=\left\{\left(\xi, w_{1}, w_{2}\right) \in S^{10} \mid \operatorname{Re}\left(w_{2}\right)=0\right\} .
$$

Then, in the sense of the last paragraph in Section [2.1, $\Sigma^{n}=D^{n} \cup_{\hat{\Theta}_{n}} D^{n}$, for $n=8,10$.

To obtain $\Theta_{10}$, one must replace $f_{10}$ by

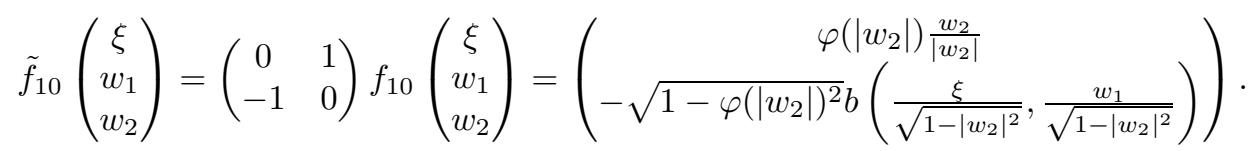

$\tilde{f}_{10}$ is equivariantly homotopic to $f_{10}$, since multiplication by the standard $S O(2) \subset$ $S p(2)$ commutes with (2.1). For simplicity, following Remark 2.2 we already replaced $\varphi$ in the final formulas.

An application of Theorem 4.6 is an explicit isotopy from the square of $\hat{\Theta}_{8}$ to the identity. This is produced by means of an equivariant homotopy from $\Theta_{8}$ to $\Theta_{8}^{-1}$, realizing the desired isotopy via the reentrance procedure.

Let $\alpha(t)=\cos t i+\sin t j$, where $i$ and $j$ are the usual unitary quaternions. We see that $w_{1} \alpha(t) \bar{w}_{1} \in \operatorname{Im} \mathbb{H}$ for every $t$ and that $\alpha(\pi)=-i$. Define

$$
\Theta_{8, t}\left(w_{1}, w_{2}\right)=\frac{w_{2}}{\left|w_{2}\right|} e^{\pi w_{1} \alpha(t) \bar{w}_{1}} \frac{\bar{w}_{2}}{\left|w_{2}\right|} .
$$

Since $\Theta_{8, t}$ is conjugation equivariant for all $t$, it defines an equivariant isotopy $\hat{\Theta}_{8, t} \hat{\Theta}_{8}=\widehat{\Theta_{8} \Theta_{8, t}}$, from $\hat{\Theta}_{8}^{2}$ (when $t=0$ ) to the identity (when $t=\pi$ ).

According to Remark 2.2 and Section 3.1, the possible lack of differentiability in $\Theta_{8}$ and $\Theta_{10}$ does not influence the present argument. A smooth approximation guarantees the result.

\section{A Gromoll-Meyer construction for Kervaire manifolds}

There is another family of manifolds admitting a description similar to $\Sigma^{8}$ and $\Sigma^{10}$. This is the well-known family of Kervaire manifolds that includes infinitely many exotic spheres (see Kos92]).

For the construction, we first present a rather non-interesting $\star$-action on the classical frame bundle $O(n) \cdots O(n+1) \rightarrow S^{n}$, and then pull it back to get a special $O(n)-O(n)$ bundle $L_{n} \rightarrow S^{2 n-1}$ whose quotient is the Kervaire $(2 n-1)$-manifold.

Consider $O(n)<O(n+1)$ as the subgroup of matrices with 1 in the upper left entry. This subgroup defines two commuting actions on $O(n+1)$ via left and right multiplication. The map $p r_{n}: O(n+1) \rightarrow S^{n}$, which sends a matrix to its first column, defines a principal bundle with the right multiplication. This bundle becomes a special $O(n)-O(n)$ bundle if we choose the left multiplication as its $\star$ action, thus endowing $S^{n}$ with the standard linear $O(n)$-action that fixes the vector 
$e_{0}=(1,0, \ldots, 0)$. Observe that the quotient by both right and left multiplications is equivariantly diffeomorphic since inversion in $O(n+1)$ sends one action to the other.

Consider $S^{2 n-1}$ as the unit sphere in $\mathbb{R}^{n} \times \mathbb{R}^{n}$ and define $J \tau: S^{2 n-1} \rightarrow S^{n}$ as

$$
J \tau\left(x_{1}, x_{2}\right)=\exp \left(\pi \tau\left(\frac{x_{2}}{\left|x_{2}\right|}\right) x_{1}\right),
$$

where $\exp (v)=\cos (|v|) e_{0}+\sin (|v|) v /|v|$ is the geodesic exponential map based on $e_{0} . J \tau$ is equivariant with respect to the standard biaxial action of $O(n)$ on $S^{2 n-1}$,

$$
q\left(x_{1}, x_{2}\right)=\left(q x_{1}, q x_{2}\right),
$$

and the $O(n)$ action we defined in $S^{n}$.

We define $L_{n}=(J \tau)^{*} O(n+1) \rightarrow S^{2 n-1}$ as the special $O(n)-O(n)$ bundle induced from $p r_{n}$, as we did for $E^{11}$ and $E^{13}$ with $p r$, via (2.4).

The $O(n)-O(n)$ bundle $p r_{n}$ can be trivialized (satisfying (3.2)) on the hemispheres defined by $\left\langle x, e_{0}\right\rangle \geq 0$ and $\left\langle x, e_{0}\right\rangle \leq 0$. On the other hand, the preimage of these hemispheres are the subsets

$$
\begin{aligned}
& D^{n} \times S^{n-1}=\left\{\left(x_{1}, x_{2}\right) \in S^{2 n-1}|| x_{1} \mid \leq 1 / 2\right\}, \\
& S^{n-1} \times D^{n}=\left\{\left(x_{1}, x_{2}\right) \in S^{2 n-1}|| x_{1} \mid \geq 1 / 2\right\},
\end{aligned}
$$

which intersect on the preimage of the equator $\left\langle x, e_{0}\right\rangle=0$, inducing a plumbing-like decomposition on $S^{2 n-1}$.

Associated to these trivializations on $p r_{n}$, we have the transition function $\tau$ : $S^{n-1} \rightarrow O(n)$ defined (as a linear map applied on $v \in \mathbb{R}^{n}$ ) by

$$
\tau(x) v=2\langle x, v\rangle x-v .
$$

It satisfies (3.1) if we endow $S^{n-1}$ with the standard linear $O(n)$-action.

Just as in (3.14) - 3.15), the transition function $\theta: S^{n-1} \times S^{n-1} \rightarrow O(n)$ related to (5.2), (5.3) is the composition $\theta\left(x_{1}, x_{2}\right)=\tau\left(J \tau\left(x_{1}, x_{2}\right)\right)$. Noticing that $\left|x_{1}\right|=1 / 2$, we have,

$$
\begin{aligned}
\tau\left(J \tau\left(x_{1}, x_{2}\right)\right) & =\tau\left(\exp \left(\pi \tau\left(\frac{x_{2}}{\left|x_{2}\right|}\right) x_{1}\right)\right)=\tau\left(\tau\left(\frac{x_{2}}{\left|x_{2}\right|}\right) \frac{x_{1}}{\left|x_{1}\right|}\right) \\
& =\tau\left(\frac{x_{2}}{\left|x_{2}\right|}\right) \tau\left(\frac{x_{1}}{\left|x_{1}\right|}\right) \tau\left(\frac{x_{2}}{\left|x_{2}\right|}\right)^{-1},
\end{aligned}
$$

from the equivariance of $\tau$. Since the map $\left(x_{1}, x_{2}\right) \mapsto \tau\left(x_{2}\right)$ equivariantly extends to $D^{n} \times S^{n-1}, L_{n}$ is equivariantly isomorphic to the bundle with transition function $r\left(x_{1}, x_{2}\right)=\tau\left(x_{1}\right) \tau\left(x_{2}\right)^{-1}$. Going over the proofs of Theorem 3.2 and its corollary, we conclude that the quotient by the resulting $\star$-action is diffeomorphic to the plumbing

$$
K^{2 n-1}=D^{n} \times S^{n-1} \cup_{g_{\tau}^{-1} f_{\tau}} S^{n-1} \times D^{n},
$$

where $f_{\tau}\left(x_{1}, x_{2}\right)=\left(x_{1}, \tau\left(x_{1}\right) x_{2}\right)$ and $g_{\tau}^{-1}\left(x_{1}, x_{2}\right)=\left(\tau\left(x_{2}\right)^{-1} x_{1}, x_{2}\right)$. According to [Bre72, Section V.8], this manifold is the Kervaire manifold of dimension $2 n-1$, which is homeomorphic to a sphere if $n$ is odd, but not diffeomorphic if, for instance, $n \neq 2^{j}-1([$ Kos92, Section X.7]).

As a last remark, we observe that the biaxial $O(n)$-action on $S^{2 n-1}$ has no fixed points. However, when $n=2 k+1$, we can reduce $p r_{n}$ to the analogous $U(k)-U(k)$ bundle defined $\left.p r_{n}\right|_{U(k+1)}: U(k+1) \rightarrow S^{2 k+1}$. This new bundle induces a biaxial 
$U(k)$-action on $S^{4 k+1}$ with many fixed points. This puts all Kervaire spheres in the context of the application in Section 4 which can be used to obtain explicit clutching diffeomorphism using the formulas in [P̈̈04].

\section{ACKNOWLEDGEMEnTs}

Part of this work is in the author's Ph.D. thesis done under the supervision of A. Rigas and C. Durán. The author would like to thank them and the anonymous referee, whose comments vastly improved the presentation of this paper.

\section{REFERENCES}

[ADPR07] U. Abresch, C. Durán, T. Püttmann, and A. Rigas, Wiedersehen metrics and exotic involutions of Euclidean spheres, J. Reine Angew. Math. 605 (2007), 1-21, DOI 10.1515/CRELLE.2007.025. MR2338125(2008e:57035)

[Bes78] Arthur L. Besse, Manifolds all of whose geodesics are closed, Ergebnisse der Mathematik und ihrer Grenzgebiete [Results in Mathematics and Related Areas], vol. 93, Springer-Verlag, Berlin-New York, 1978. With appendices by D. B. A. Epstein, J.-P. Bourguignon, L. Bérard-Bergery, M. Berger and J. L. Kazdan. MR496885 (80c:53044)

[Bre72] Glen E. Bredon, Introduction to compact transformation groups, Academic Press, New York-London, 1972. Pure and Applied Mathematics, Vol. 46. MR0413144 (54 \#1265)

[Dav82] Michael W. Davis, Some group actions on homotopy spheres of dimension seven and fifteen, Amer. J. Math. 104 (1982), no. 1, 59-90, DOI 10.2307/2374068. MR648481 (83g:57027)

[DMR04] C. E. Durán, A. Mendoza, and A. Rigas, Blakers-Massey elements and exotic diffeomorphisms of $S^{6}$ and $S^{14}$ via geodesics, Trans. Amer. Math. Soc. 356 (2004), no. 12, 5025-5043, DOI 10.1090/S0002-9947-04-03469-5. MR.2084409 (2005e:53055)

[DP09] C. Durán and T. Püttmann, A minimal brieskorn 5-sphere in the Gromoll-Meyer sphere and its applications, Michigan Mathematical Journal, pages 419-451, 2009.

[DPR10] Carlos Durán, Thomas Püttmann, and A. Rigas, An infinite family of Gromoll-Meyer spheres, Arch. Math. (Basel) 95 (2010), no. 3, 269-282, DOI 10.1007/s00013-010-0161x. MR2719385 (2011i:57043)

[DR09] C. Durán and A. Rigas, Equivariant homotopy and deformations of diffeomorphisms, Differential Geom. Appl. 27 (2009), no. 2, 206-211, DOI 10.1016/j.difgeo.2008.06.018. MR2503973(2009m:58021)

[DRS10] C. E. Durán, A. Rigas, and L. D. Sperança, Bootstrapping Ad-equivariant maps, diffeomorphisms and involutions, Mat. Contemp. 35 (2008), 27-39. MR2584174 (2010m:57042)

[Dur01] Carlos E. Durán, Pointed Wiedersehen metrics on exotic spheres and diffeomorphisms of $S^{6}$, Geom. Dedicata 88 (2001), no. 1-3, 199-210, DOI 10.1023/A:1013163427655. MR1877216 (2002i:57044)

[EK62] J. Eeells and N. Kuiper, An invariant of certain smooth manifolds, Annali Mat. Pura e Appl., 60:413-443, 2962.

[GM72] D. Gromoll and W. Meyer, An exotic sphere with nonnegative curvature, Annals of Mathematics, 96:413-443, 1972.

[Kos92] A. Kosinsiski, Differentiable Manifolds, Boston, MA: Academic Press, 1992.

[Las82] R. K. Lashof, Equivariant bundles, Illinois J. Math. 26 (1982), no. 2, 257-271. MR650393 (83g:57025)

[Mil56] John Milnor, On manifolds homeomorphic to the 7-sphere, Ann. of Math. (2) 64 (1956), 399-405. MR0082103 (18,498d)

[Mil59A] John Milnor, Differentiable structures on spheres, Amer. J. Math. 81 (1959), 962-972. MR0110107 (22 \#990)

[Mil59B] J. Milnor, Differentiable manifolds which are homotopy spheres, Unpublished notes, Princeton University, 1959,

[Pü04] T. Püttmann, Some homotopy groups of the classical groups from a geometric viewpoint, Habilitationsschrift, January 2004.

[Sch72] Reinhard Schultz, Circle actions on homotopy spheres bounding plumbing manifolds, Proc. Amer. Math. Soc. 36 (1972), 297-300. MR0309138 (46 \#8248) 
[Ste99] Norman Steenrod, The topology of fibre bundles, Princeton Landmarks in Mathematics, Princeton University Press, Princeton, NJ, 1999. Reprint of the 1957 edition; Princeton Paperbacks. MR1688579 (2000a:55001)

[Wil01] Frederick Wilhelm, Exotic spheres with lots of positive curvatures, J. Geom. Anal. 11 (2001), no. 1, 161-186, DOI 10.1007/BF02921960. MR1829354 (2002c:53062)

Departamento de Matemática, UfPr, Setor de Ciências Exatas, Centro Politécnico, Caixa Postal 019081, CEP 81531-990, Curitiba, PR, Brazil

E-mail address: 1speranca@ufpr.br 